OPEN ACCESS

Edited by:

Margaret E. Bauer,

Indiana University Bloomington,

United States

Reviewed by:

Catherine M. Logue,

University of Georgia, United States

Amy Michele Grunden,

North Carolina State University,

United States

*Correspondence:

Shaowen L

lishaowen@mail.hzau.edu.cn

tThese authors have contributed equally to this work.

Received: 18 March 2018 Accepted: 05 June 2018

Published: 22 June 2018

Citation:

Li B, Huang Q, Cui A, Liu X, Hou B,

Zhang L, Liu M, Meng $X$ and LiS

(2018) Overexpression of Outer

Membrane Protein X (OmpX)

Compensates for the Effect of TolC

Inactivation on Biofilm Formation and

Curli Production in Extraintestinal

Pathogenic Escherichia coli (ExPEC).

Front. Cell. Infect. Microbiol. 8:208

doi: 10.3389/fcimb.2018.00208

\section{Overexpression of Outer Membrane Protein X (OmpX) Compensates for the Effect of TolC Inactivation on Biofilm Formation and Curli Production in Extraintestinal Pathogenic Escherichia coli (ExPEC)}

\author{
Binyou Li ${ }^{\dagger}$, Qi Huang ${ }^{\dagger}$, Ailian Cui, Xueling Liu, Bo Hou, Liyuan Zhang, Mei Liu, \\ Xianrong Meng and Shaowen $\mathrm{Li}^{*}$
}

Key Laboratory of Preventive Veterinary Medicine in Hubei Province, College of Veterinary Medicine, Huazhong Agricultural University, Wuhan, China

Our previous study showed that the inactivation of the efflux pump TolC could abolish biofilm formation and curli production of extraintestinal pathogenic Escherichia coli (ExPEC) strain PPECC42 under hyper-osmotic conditions. In this study we investigated the role of OmpX in biofilm formation and curli production of ExPEC PPECC42. Our data showed that OmpX disruption or overexpression didn't significantly affect the biofilm formation and curli production of the wild-type strain. However, in the tolC-deleted mutant, overexpressing OmpX suppressed the effect of TolC inactivation on EXPEC biofilm formation and curli production under hyper-osmotic growth conditions. Real-time qRT-PCR confirmed that OmpX overexpression affected curli production by regulating the transcription of the curli biosynthesis-related genes in the $\Delta$ to/C strain. Our findings suggest that $\mathrm{OmpX}$ is involved in biofilm formation and curli production.

Keywords: extraintestinal pathogenic Escherichia coli, outer membrane protein X, TolC, biofilm formation, curli production

\section{INTRODUCTION}

Extraintestinal pathogenic Escherichia coli (ExPEC) are a sub group of pathogenic E. coli strains causing a variety of infections and diseases at extraintestinal sites in humans and animals, which are typically characterized by multi-organ infections including urinary tract infections, meningitis, polyserositis, and septicemia (Johnson and Russo, 2005; Smith et al., 2007; Köhler and Dobrindt, 2011; Manges and Johnson, 2012; Mitchell et al., 2015). Recently, ExPEC strains were frequently isolated from clinical samples of pigs (Ding et al., 2012). Moreover, they were widely found in pork, retail chicken, beef, and ready-to-eat foods, which pose a potential threat to public health (Lyhs et al., 2012; Aslam et al., 2014; Mitchell et al., 2015).

Biofilms are defined as structured communities of bacterial cells enclosed in a self-produced polymeric matrix and adhered to an inert or living surface (Costerton et al., 1999). Biofilms can increase bacterial resistance to external environmental stresses such as exposure to antimicrobials, 
antiseptics, desiccation, and extremes of temperature, which can help the bacteria to survive in different hostile environments (Steenackers et al., 2012; de La Fuente-Núñez et al., 2013). Biofilms can also serve as a physical barrier to protect bacteria from eradication by the host immune defense system (Donlan and Costerton, 2002), and are considered to be an important virulence factor in ExPEC (Magistro et al., 2015). Therefore, exploration of the role of biofilm formation in pathogenesis and virulence is important in investigating factors that influence biofilm formation and for addressing means to prevent or inhibit/eradicate biofilms in the production and human settings.

Biofilm formation is a complicated process and controlled by complex networks in E. coli. Bacterial active motility achieved by flagella movement increases the chance of bacteria to interact with surfaces (Donlan, 2002). Once attached to the surface, bacterial fimbriae, mainly type 1 fimbriae and curli, and adhesins, such as Ag43, promote adhesion to surfaces (Danese et al., 2000; Holden and Gally, 2004). After initial attachment, bacteria produce a number of extracellular components constituting the biofilm matrix, mainly composed of amyloid curli fibers and cellulose. Biofilm formation involves considerable regulations at transcriptional as well as post-transcriptional levels. Environmental changes affecting biofilm formation are sensed by several two-component systems, including $\mathrm{CpxA} / \mathrm{R}, \mathrm{RcsC} / \mathrm{D} / \mathrm{B}$, and EnvZ/OmpR, which mediate transcriptional regulations of genes involving outer membrane protein production, flagellar synthesis, curli expression etc. (Prigent-Combaret et al., 2001; Otto and Silhavy, 2002; Ferrières and Clarke, 2003). The global transcription factors, including RpoS, H-NS, and BolA, and the master regulators for curli fimbriae expression, CsgD, are believed to play dominant roles in the transcriptional regulation of biofilm formation (reviewed in Mika and Hengge, 2014). Small molecules, including the well-known second messenger c-di-GMP, the alarmone ppGpp, N-acetylglucosamine-6$\mathrm{P}$ (GlcNAc-6P), and the auto-inducer-2 (AI-2), function as post-transcriptional factors regulating bacterial biofilm formation. (Balzer and Mclean, 2002; Ren et al., 2004; Barnhart et al., 2006; González Barrios et al., 2006; Jenal and Malone, 2006).

Curli fimbriae play important roles in the irreversible adhesion stage of $E$. coli biofilm formation to form self-produced extracellular matrix, enhance initial cell-cell interactions, and adhesion to biotic and abiotic surfaces, and eventually promote biofilm formation (Austin et al., 1998; White et al., 2003; Barnhart and Chapman, 2006; Beloin et al., 2008). Curli biosynthesisrelated genes in $E$. coli cluster in two divergent operons: $c s g D E F G$ and $\operatorname{csg} B A C$ (Van Houdt and Michiels, 2005). The csgBAC operon encodes the major structural subunit CsgA and the core protein CsgB (Hammar et al., 1995, 1996). The csgDEFG operon encodes four accessory proteins which facilitate translocation of the curli subunits across the outer membrane and contribute to curli assembly and stability, in which CsgD is an essential positive transcriptional regulator of the curli regulatory network. The expression of curli biosynthesis-related genes is also regulated by several other transcription factors, such as RpoS and H-NS (Austin et al., 1998; Chapman et al., 2002).
Our previous study showed that inactivation of TolC compromised the ability of biofilm formation and curli production in porcine ExPEC strain PPECC42 in response to hyper-osmotic conditions (Hou et al., 2014). TolC is the major channel for drug efflux across the outer membrane of E. coli, and is well-known for its involvement in the transportation of various types of chemicals, including antibiotics, disinfectants, and metabolic products, leading to bacterial antimicrobial resistance (Zgurskaya et al., 2011). Other than a drug efflux pump, TolC has also been reported to be involved in biofilm formation in Actinobacillus pleuropneumoniae, a porcine respiratory tract pathogen (Li et al., 2016). However, the underlying mechanism of how TolC affects biofilm formation remains largely unknown. In order to explain how TolC affected biofilm formation, we carried out a comparative proteomic analysis which showed that the expression of outer membrane protein X (OmpX) was abolished in the $\triangle$ tolC strain detected by using SDS-PAGE combined with MALDI-TOF mass-spectrometry (data not shown).

OmpX and its homologs have been identified in many Gram-negative bacteria, such as Enterobacter cloacae (OmpX) (Stoorvogel et al., 1991), Salmonella enterica serovar Typhimurium (PagC, Rck) (Heffernan et al., 1992), Yersinia spp. (Ail) (Kolodziejek et al., 2010), E. coli (OmpX, Lom) (Mecsas et al., 1995), and Klebsiella pneumoniae (OmpK17) (Climent et al., 1997). These proteins are of low molecular weight (from 15 to $18 \mathrm{kDa}$ ) and fold in an eight- $\beta$-barrel structure with membrane-spanning domains that protrude from the cell surface. They are involved in considerable number of physiological processes including binding external proteins, participating in channeling, antibiotic resistance, signal transduction, invasion, survival in macrophages, internalization in epithelial cells and virulence (Stoorvogel et al., 1991; Vogt and Schulz, 1999; Miller et al., 2001; Otto and Hermansson, 2004; Kolodziejek et al., 2010; Meng et al., 2016). OmpX overproduction was considered to be a bacterial adaptive response toward environmental stresses (Dupont et al., 2007). However, the role of $\mathrm{OmpX}$ in bacterial biofilm formation remains unclear. The present study investigated the role of OmpX in ExPEC biofilm formation using both gene deletion and overexpression assays. Our data showed that OmpX overexpression suppressed the effect of tolC inactivation on ExPEC biofilm formation and curli production in response to the hyper-osmotic stress.

\section{MATERIALS AND METHODS}

\section{Strain Construction}

Strains, plasmids, and primers used in this work are listed in Table 1. The wild-type (WT) ExPEC strain PPECC42 was isolated from the lung of a diseased pig in China in 2006. Its complete genome sequence has been submitted to NCBI (Genbank Accession No. NZ_CM003707.1). An isogenic ompXdeleted strain of ExPEC PPECC42, in which a 345 bp fragment was deleted within the ompX open reading frame (ORF), was constructed as described previously (Meng et al., 2016). To construct the strains overexpressing OmpX, the plasmid pHSG::ompX containing the full-length ompX gene of ExPEC 
TABLE 1 | Strains, plasmids, and primers used in this work.

\begin{tabular}{|c|c|c|}
\hline & Description or sequence & Source \\
\hline \multicolumn{3}{|l|}{ STRAIN } \\
\hline EXPEC strain PPECC42 & Wild-type (WT), porcine origin, $\mathrm{Cm}^{\mathrm{S}}$ & Hou et al., 2014 \\
\hline$\Delta o m p X$ & $\begin{array}{l}\text { Mutant with a 516-bp fragment deleted from the whole ORF } \\
\text { of the ompX gene in PPECC42, } \mathrm{Cm}^{S}\end{array}$ & Meng et al., 2016 \\
\hline WT::ompX & PPECC42 strain carrying plasmid pHSG::ompX, $\mathrm{Cm}^{R}$ & This study \\
\hline$\times 7213$ & $\begin{array}{l}\text { Thi-1 thr-1 leuB6 fhuA21 lacY1 glnV44 } \triangle \text { asdA4 recA1 RP4 } \\
\text { 2-Tc::Mu[ } \lambda \text { pir] KmR }\end{array}$ & Hou et al., 2014 \\
\hline $\mathrm{DH} 5 \alpha$ & $\begin{array}{l}\text { F-, } \phi 80 \text { dlacZ } \Delta M 15, \Delta(\text { lacZYA-argF) U169, deoR, recA1, } \\
\left.\text { endA1, hsdR17 (rk- }{ }^{-} \text {mk }^{+}\right) \text {, phoA,supE44, } \\
\lambda^{-} \text {,thi-1,gyrA96,relA1 }\end{array}$ & Takara Bio \\
\hline pHSG::ompX & ori lacZ $\mathrm{Cm}^{\mathrm{R}}$ pHSG396 with the full-length ompX gene & Meng et al., 2016 \\
\hline \multicolumn{3}{|l|}{ PRIMER } \\
\hline$o m p X_{F}$ & ACCTGAAATACCGCTATGAA & \\
\hline$o m p X_{R}$ & TCAGTGGTCTGGAATTTACC & \\
\hline $\operatorname{csg} D_{F}$ & CCCGTACCGCGACATTG & \\
\hline $\operatorname{csg} D_{R}$ & ACGTTCTTGATCCTCCATGGA & \\
\hline $\operatorname{csg} B_{F}$ & CATAATTGGTCAAGCTGGGACTAA & \\
\hline $\operatorname{csg} B_{R}$ & GCAACAACCGCCAAAAGTTT & \\
\hline$G A P D H_{F}$ & ACTTACGAGCAGATCAAAGC & \\
\hline$G A P D H_{R}$ & AGTTTCACGAAGTTGTCGTT & \\
\hline
\end{tabular}

strain PPECC42 (Meng et al., 2016) was electroporated into each strain needed. Clones were selected on LB agar plates containing chloramphenicol. The primers used in this work are shown in Table 1. All strains were grown either in Lysogeny broth (LB) or in M9 or 1/2 M9 minimal medium. When necessary, ampicillin or chloramphenicol was used at 100 and $25 \mu \mathrm{g} / \mathrm{ml}$, respectively.

\section{Determination of Growth Kinetics}

Fresh LB medium, M9 or 1/2 M9 minimal medium was inoculated with a 1:100 dilution of overnight cultures and incubated at $37^{\circ} \mathrm{C}$ under shaking at $200 \mathrm{rpm}$. Samples were collected every hour and the optical densities were measured at $600 \mathrm{~nm}\left(\mathrm{OD}_{600}\right)$ using a BioPhotometer (Eppendorf, Hamburg, Germany). The data were obtained from three independent experiments, with each having three biological replicates.

\section{Crystal Violet Biofilm Assay}

Biofilm formation was evaluated using crystal violet assay as described previously (Stepanovic et al., 2000). Briefly, the overnight grown bacterial culture was diluted 1:100 in each indicated medium in a 96-well microtiter plate (Nunc, Denmark) and incubated at $28^{\circ} \mathrm{C}$. The wells without bacterial inoculation were taken as the negative control. After $120 \mathrm{~h}$, the cells were removed, and the wells were washed five times with sterile distilled water. The wells were stained with $125 \mu \mathrm{l}$ of $1.0 \%(\mathrm{w} / \mathrm{v})$ crystal violet for $15 \mathrm{~min}$, washed with sterile distilled water, and the biofilm was then dissolved with $150 \mu \mathrm{l}$ of $33 \%$ (v/v) glacial acetic acid. The optical density of each well was measured at $630 \mathrm{~nm}\left(\mathrm{OD}_{630}\right)$ using a Universal Microplate Reader (Bio-Tek, Winooski, USA). All assays were performed with 12 replicates. The cutoff OD (ODc) was defined as three times standard deviation above the mean OD of the negative control.

\section{Visualization of Curli Fimbriae}

Curli production was assessed by morphological examination of colonies grown on M9 or 1/2 M9 agar plates containing $40 \mu \mathrm{g} / \mathrm{mL}$ Congo red (Amresco, Ohio, USA) and $20 \mu \mathrm{g} / \mathrm{mL}$ Coomassie brilliant blue (Solarbio, Beijing, China) as described previously (Lloyd et al., 2012). Briefly, $1 \mu \mathrm{L}$ of the overnight grown culture of each strain was spotted onto the Congo red plate and incubated at $28^{\circ} \mathrm{C}$ for $120 \mathrm{~h}$. The morphology of each bacterial spot was observed and imaged. The experiments were carried out in triplicate.

\section{Real-Time Quantitative Reverse Transcription Polymerase Chain Reaction (qRT-PCR)}

Total RNAs were extracted using the RNeasy Mini Kit (OMEGA, Minnesota, USA) from the cells of each strain cultured in different conditions. cDNA was reverse transcribed from $2 \mu \mathrm{g}$ of RNA using HiScript TM first strand cDNA synthesis kit 
(Vazyme, New Jersey, USA), and used as the template for qRTPCR with AceQ ${ }^{\mathrm{TM}}$ qPCR SYBR Green Master Mix Kit (Vazyme, New Jersey, USA) using a Bio-Rad detection system (Bio-Rad, California, USA). The expression of the target genes in each strain was normalized to that of the house-keeping gene GAPDH using the delta-delta threshold cycle $(\Delta \Delta \mathrm{CT})$ method (Viveiros et al., 2007), with mutants Ct values representing the fold change relative to that of the $\mathrm{WT}$ strain, which was set at 1 . Comparative qRT-PCR was used to determine the average expression from four replicate wells. The assays were repeated using RNAs harvested from independent cultures of each strain in triplicate.

\section{Statistical Analysis}

Statistical analyses were performed using GraphPad prism version 5.0 software (GraphPadPrism Software, San Diego, CA). Statistical difference was calculated using the One-way ANOVA test and considered significant at a $p<0.05$.

\section{RESULTS}

\section{Disruption or Overexpression of OmpX Does not Affect Cell Growth}

OmpX overexpression was achieved by introducing plasmid pHSG::ompX into each strain. The expression level of OmpX in each strain was analyzed by using real-time qRT-PCR. As shown in Figure 1A, at each indicated time-point $(24,72$, and $120 \mathrm{~h})$ of growth, expression of OmpX was not detected in the $\Delta o m p X$ mutant, but was detected in the $\Delta$ tolC::ompX and WT::ompX strains, suggesting that the strains construction was successful. The growth curves of each strain were determined in LB medium, M9 minimal medium, and 1/2 M9 minimal medium, and the results showed that all the strains presented a similar growth pattern to the WT strain (Figures 1B-D), indicating that OmpX inactivation and overexpression do not significantly affect the growth of ExPEC.

\section{Deletion or Overexpression of OmpX Does Not Significantly Affect the Biofilm Formation of the Wild-Type Expec}

We next investigated the effect of OmpX deletion and overexpression on biofilm formation of ExPEC. Cells were grown in $1 / 2 \mathrm{M} 9$ or $\mathrm{M} 9$ medium at $28^{\circ} \mathrm{C}$ for $120 \mathrm{~h}$ and the biofilm formation was measured using crystal violet biofilm assay. As shown in Figures 2A,B, the wild-type strain with overexpressed OmpX (indicated as WT::ompX) or deleted $\operatorname{ompX}$ (indicated as ompX) showed a comparable level of biofilm formation to the wild-type strain (WT), indicating the deletion or overexpression of OmpX alone does not affect the biofilm formation of the wild-type ExPEC.

\section{Overexpression of OmpX Restored the Biofilm Formation of the $\Delta$ to/C Strain Under Hyper-Osmotic Conditions}

Our previous study revealed that the disruption of tolC led to decreased biofilm formation when the cells were grown in M9 medium (Hou et al., 2014). We next tested whether OmpX has a similar role on the tolC deletion strain and influencing its ability to form biofilms. As shown in Figure 2A, the wildtype strain showed a strong ability of biofilm formation while the $\Delta$ tolC strain almost lost the ability of biofilm formation, which was consistent with our previous study (Hou et al., 2014). In contrast, overexpressing OmpX in the $\Delta$ tolC mutant (indicated as $\Delta$ tolC::ompX) restored the ability to form biofilm to a similar level of the wild-type strain (Figure 2A), suggesting that the overproduction of OmpX compensated for the defective biofilm formation caused by tolC inactivation in the M9 medium. It was seen that in 1/2 M9 medium, all the strains formed strong biofilms (Figure 2B). Considering the difference between M9 and 1/2 M9 media in osmolarity, we speculated that the influence of OmpX overexpression on biofilm formation might be related to external osmotic response. Therefore, the biofilm formation of the $\Delta$ tolC strain with or without overexpressed OmpX was measured in sucrose- and $\mathrm{NaCl}$ - induced hyperosmotic conditions, respectively. As shown in Figures 2C,D in both conditions, the overexpression of OmpX was able to restore the ability of biofilm formation of the $\Delta t o l C$ strain. The biofilm formation of the $\Delta o m p X$ strain and the wild-type strain overexpressing OmpX was not affected under these conditions. The above results strongly suggest that OmpX overexpression compensates for the defective biofilm formation induced by hyper-osmotic stresses of the $\Delta$ tolC strain.

\section{OmpX Overexpression Compensates for the Defective Curli Production of the $\Delta t o / C$ Mutant}

Our previous study showed that the inactivation of tolC reduced the curli production in hyper-osmotic conditions (Hou et al., 2014). So we next investigated whether OmpX overexpression also suppressed the curli production of the $\Delta$ tolC mutant. A Congo red (CR) assay was used to test the curli production. As shown in Figure $3 \mathrm{~A}$, all of the four strains, WT, $\Delta$ tolC, $\Delta$ tolC::ompX, and WT::ompX, presented a rdar (red, dry, and rough) morphology on 1/2 M9-CR agar plates, indicating normal curli production. However, in M9 medium and under $0.06 \mathrm{M}$ $\mathrm{NaCl}$ - or $0.8 \%$ sucrose-induced hyper-osmotic conditions, in contrast with the wild-type strain that still presented a rdar morphology, the $\Delta$ tolC strain showed a significantly whiter morphology, which was consistent with our previous study that the disruption of tolC reduced the curli production. Intriguingly, overproducing OmpX restored the morphology of the cells of $\Delta$ tolC strain to a similar level to the WT strain. This result suggests that OmpX overexpression suppresses the reduced curli production resulted from tolC deletion under hyper-osmotic stresses.

The transcription levels of two separate curli biosynthesisrelated genes, $\operatorname{csg} D$ and $\operatorname{csg} B$, were further tested in the four strains grown in the M9 medium. As shown in Figure 3B, at each indicated time point of growth, compared with the WT strain, the $\Delta$ tolC mutant showed a significantly down-regulated expression of both of $\operatorname{csg} D$ and $\operatorname{csgB}$ genes. In contrast, the $\Delta$ tolC::ompX strain exhibited a much higher expression level of sboth genes than the $\triangle$ tolC strain. The results further suggested that the 

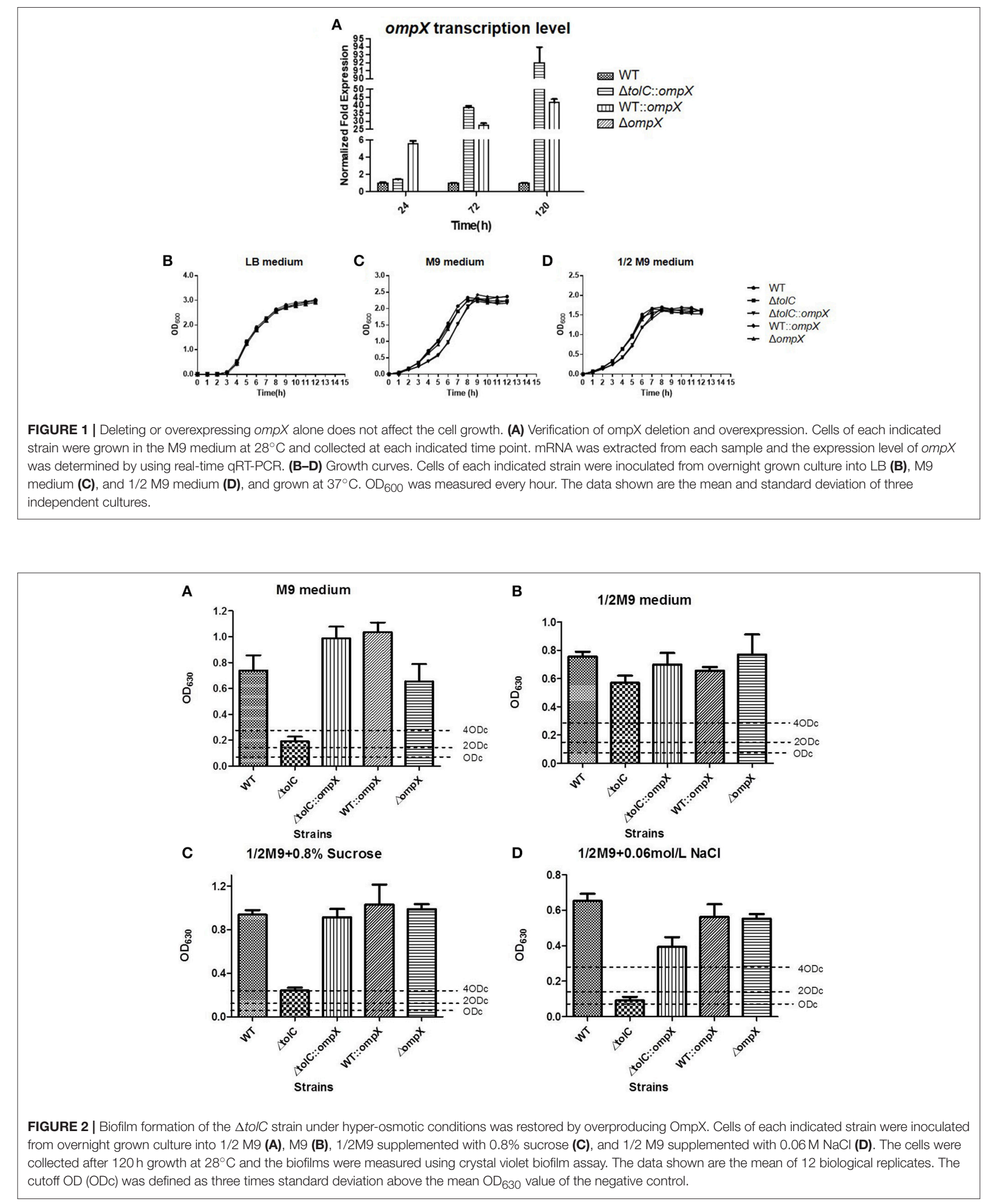


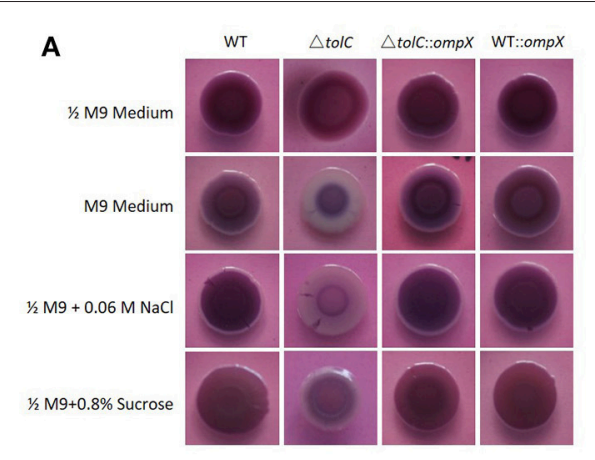

B
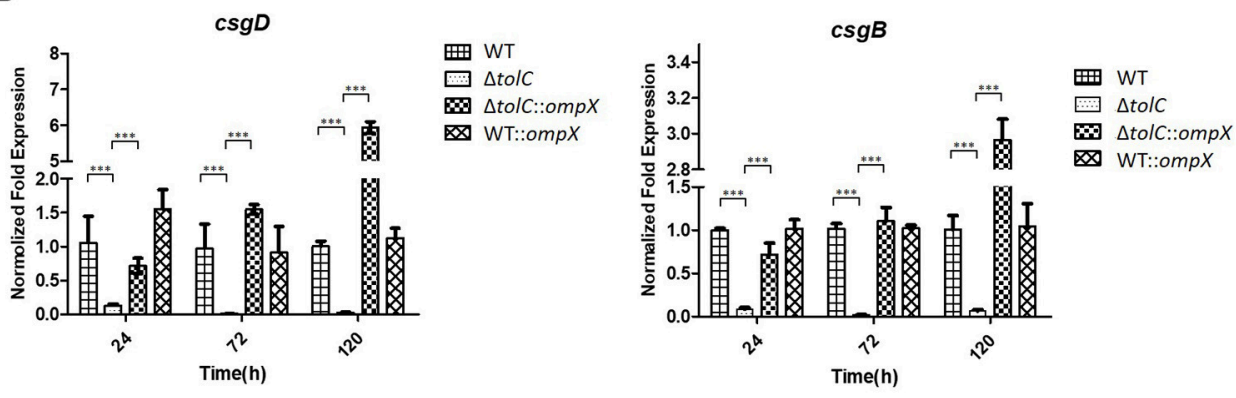

FIGURE 3 | ompX overexpression restored the curli production of the $\Delta$ to/C strain under hyper-osmotic conditions. (A) Congo red (CR) assay analysis of curli production. One microliter of overnight grown cells of each indicated stain were spotted to the agar plates containing $40 \mu \mathrm{g} / \mathrm{ml}$ Congo red, which were incubated at $28^{\circ} \mathrm{C}$ for $120 \mathrm{~h}$. (B) Transcription analysis of csgD and csgB. The cells of each indicated strain were grown in the $\mathrm{M} 9 \mathrm{medium}$ at $28^{\circ} \mathrm{C}$ and the cells were collected at each indicated timepoint. RNA was extracted and the relative levels were determined using real-time qRT-PCR. The data shown are the mean of three biological replicates. The error bars represented the standard deviations. Significant differences were determined using the One-way Anova test. ${ }^{\star \star \star} P<0.001$.

regulation of OmpX overexpression on curli production is due to its effect on the transcription of the curli biosynthesis-related genes.

\section{DISCUSSION}

Biofilms can increase bacterial resistance to external environmental stresses, and help the bacteria survive in different hostile environments (Steenackers et al., 2012; de La Fuente-Núñez et al., 2013). Efflux pump protein TolC is involved in the transportation of various types of chemicals, including antibiotics, disinfectants, and metabolic products. Our previous study confirmed that inactivation of TolC compromised ExPEC biofilm formation and curli production in response to high osmolarity (Hou et al., 2014). Our preliminary proteomic data showed that the OmpX protein was not detected in the $\triangle$ tolC mutant, indicating potential interplays between TolC and OmpX. Hence, this study was designed to explore the role of OmpX in ExPEC biofilm formation and curli production, especially under hyper-osmotic conditions, and to determine whether OmpX mediated the effect of TolC inactivation on ExPEC biofilm formation.

Our data showed that the disruption or overexpression of OmpX alone did not have any effect on biofilm formation; however, in the $\Delta$ tolC background OmpX overexpression significantly suppressed the inhibited biofilm formation and curli production under hyper-osmotic conditions. TolC is able to regulate many proteins and interact with other outer membrane proteins in E. coli under different concentrations of glucose (Yang et al., 2011). The deletion of tolC increased the transcription of ompC and micF under high osmotic conditions, whereafter reduced the amount of OmpF (Misra and Reeves, 1987). OmpX overproduction resulted in a decrease in expression of a classical porin Omp36 in E. aerogenes (Dupont et al., 2004). Overproduction of OmpX and downregulation of porins were considered to be a bacterial adaptive response toward environmental stresses (Dupont et al., 2007). OmpX overexpression could also increase the activity of $\sigma \mathrm{E}$, an important envelope stress response factor in E. coli (Otto and Hermansson, 2004; Pletzer et al., 2015).

Therefore, this study suggests that TolC and OmpX play important roles in responding to and resisting osmotic stresses. When confronting hyper-osmotic conditions, the bacterial cells lacking TolC became unable to carry out proper osmolarity regulations, therefore were sensitive to these stresses. Under this circumstance, OmpX overexpression may modify the expression of $\mathrm{NaCl}$ or sucrose transportation related proteins or signal pathways, increase the efflux or decrease the influx of $\mathrm{NaCl}$ or sucrose across the outer membrane, and thus relieve the hyperosmolarity stresses and regulate curli production and biofilm formation. The current study provides a new insight into the role of OmpX in bacterial biofilm formation and environmental osmotic adaptation. 


\section{AUTHOR CONTRIBUTIONS}

$\mathrm{BL}, \mathrm{QH}, \mathrm{AC}, \mathrm{XL}, \mathrm{BH}$, and $\mathrm{LZ}$ performed research. $\mathrm{QH}, \mathrm{BL}, \mathrm{ML}$, $\mathrm{XM}$, and SL designed research and analyzed the data. $\mathrm{BL}, \mathrm{QH}$, and SL wrote the paper.

\section{REFERENCES}

Aslam, M., Toufeer, M., Narvaez Bravo, C., Lai, V., Rempel, H., Manges, A., et al. (2014). Characterization of extraintestinal pathogenic Escherichia coli isolated from retail poultry meats from Alberta, Canada. Int. J. Food Microbiol. 177, 49-56. doi: 10.1016/j.ijfoodmicro.2014.02.006

Austin, J. W., Sanders, G., Kay, W. W., and Collinson, S. K. (1998). Thin aggregative fimbriae enhance Salmonella enteritidis biofilm formation. FEMS Microbiol. Lett. 162, 295-301. doi: 10.1111/j.1574-6968.1998.tb13012.x

Balzer, G. J., and Mclean, R. J. (2002). The stringent response genes relA and spoT are important for Escherichia coli biofilms under slow-growth conditions. Can. J. Microbiol. 48, 675-680. doi: 10.1139/w02-060

Barnhart, M. M., and Chapman, M. R. (2006). Curli biogenesis and function. Annu. Rev. Microbiol. 60, 131-147. doi: 10.1146/annurev.micro.60.080805.142106

Barnhart, M. M., Lynem, J., and Chapman, M. R.,(2006). GlcNAc-6P levels modulate the expression of Curli fibers by Escherichia coli. J. Bacteriol. 188, 5212-5219. doi: 10.1128/JB.00234-06

Beloin, C., Roux, A., and Ghigo, J. M. (2008). Escherichia coli biofilms. Curr. Top. Microbiol. Immunol. 322, 249-289. doi: 10.1007/978-3-540-75418-3 12

Chapman, M. R., Robinson, L. S., Pinkner, J. S., Roth, R., Heuser, J., Hammar, M., et al. (2002). Role of Escherichia coli curli operons in directing amyloid fiber formation. Science 295, 851-855. doi: 10.1126/science.1067484

Climent, N., Ferrer, S., Rubires, X., Merino, S., Tomas, J. M., and Regue, M. (1997). Molecular characterization of a $17-\mathrm{kDa}$ outer-membrane protein from Klebsiella pneumoniae. Res. Microbiol. 148, 133-143. doi: 10.1016/S0923-2508(97)87644-9

Costerton, J. W., Stewart, P. S., and Greenberg, E. P. (1999). Bacterial biofilms: a common cause of persistent infections. Science 284, 1318-1322. doi: $10.1126 /$ science.284.5418.1318

Danese, P. N., Pratt, L. A., Dove, S. L., and Kolter, R. (2000). The outer membrane protein, antigen 43, mediates cell-to-cell interactions within Escherichia coli biofilms. Mol. Microbiol. 37, 424-432. doi: 10.1046/j.1365-2958.2000.02008.x

de La Fuente-Núñez, C., Reffuveille, F., Fernandez, L., and Hancock, R. E. (2013). Bacterial biofilm development as a multicellular adaptation: antibiotic resistance and new therapeutic strategies. Curr. Opin. Microbiol. 16, 580-589. doi: 10.1016/j.mib.2013.06.013

Ding, Y., Tang, X., Lu, P., Wu, B., Xu, Z., Liu, W., et al. (2012). Clonal analysis and virulent traits of pathogenic extraintestinal Escherichia coli isolates from swine in China. BMC Vet. Res. 8:140. doi: 10.1186/1746-6148-8-140

Donlan, R. M. (2002). Biofilms: microbial life on surfaces. Emerg. Infect. Dis. 8, 881-890. doi: 10.3201/eid0809.020063

Donlan, R. M., and Costerton, J. W. (2002). Biofilms: survival mechanisms of clinically relevant microorganisms. Clin. Microbiol. Rev. 15, 167-193. doi: 10.1128/CMR.15.2.167-193.2002

Dupont, M., De, E., Chollet, R., Chevalier, J., and Pages, J. M. (2004). Enterobacter aerogenes OmpX, a cation-selective channel mar- and osmo-regulated. FEBS Lett. 569, 27-30. doi: 10.1016/j.febslet.2004.05.047

Dupont, M., James, C. E., Chevalier, J., and Pages, J. M. (2007). An early response to environmental stress involves regulation of OmpX and OmpF, two enterobacterial outer membrane pore-forming proteins. Antimicrob. Agents Chemother. 51, 3190-3198. doi: 10.1128/AAC.01481-06

Ferrières, L., and Clarke, D. J. (2003). The RcsC sensor kinase is required for normal biofilm formation in Escherichia coli K-12 and controls the expression of a regulon in response to growth on a solid surface. Mol. Microbiol. 50, 1665-1682. doi: 10.1046/j.1365-2958.2003.03815.x

González Barrios, A. F., Zuo, R., Hashimoto, Y., Yang, L., Bentley, W. E., and Wood, T. K. (2006). Autoinducer 2 controls biofilm formation in Escherichia coli through a novel motility quorum-sensing regulator (MqsR, B3022). J. Bacteriol. 188, 305-316. doi: 10.1128/JB.188.1.305-316.2006

\section{ACKNOWLEDGMENTS}

This work was supported by the National Natural Science Foundation of China (grant number 31572539 to $\mathrm{SL}$ ).

Hammar, M. R., Arnqvist, A., Bian, Z., Olsen, A., and Normark, S. (1995) Expression of two csg operons is required for production of fibronectin- and Congo red-binding curli polymers in Escherichia coli K-12. Mol. Microbiol. 18, 661-670. doi: 10.1111/j.1365-2958.1995.mmi_18040661.x

Hammar, M., Bian, Z., and Normark, S. (1996). Nucleator-dependent intercellular assembly of adhesive curli organelles in Escherichia coli. Proc. Natl. Acad. Sci. U.S.A. 93, 6562-6566. doi: 10.1073/pnas.93.13.6562

Heffernan, E. J., Harwood, J., Fierer, J., and Guiney, D. (1992). The Salmonella typhimurium virulence plasmid complement resistance gene rck is homologous to a family of virulence-related outer membrane protein genes, including pagC and ail. J. Bacteriol. 174, 84-91. doi: 10.1128/jb.174.1.84-91.1992

Holden, N. J., and Gally, D. L. (2004). Switches, cross-talk and memory in Escherichia coli adherence. J. Med. Microbiol. 53, 585-593. doi: 10.1099/jmm.0.05491-0

Hou, B., Meng, X. R., Zhang, L. Y., Tan, C., Jin, H., Zhou, R., et al. (2014). TolC promotes ExPEC biofilm formation and curli production in response to medium osmolarity. Biomed Res. Int. 2014:574274. doi: 10.1155/2014/574274

Jenal, U., and Malone, J. (2006). Mechanisms of cyclic-diGMP signaling in bacteria. Annu. Rev. Genet. 40, 385-407. doi: 10.1146/annurev.genet.40.110405.090423

Johnson, J. R., and Russo, T. A. (2005). Molecular epidemiology of extraintestinal pathogenic (uropathogenic) Escherichia coli. Int. J. Med. Microbiol. 295, 383-404. doi: 10.1016/j.ijmm.2005.07.005

Köhler, C. D., and Dobrindt, U. (2011). What defines extraintestinal pathogenic Escherichia coli? Int. J. Med. Microbiol. 301, 642-647. doi: 10.1016/j.ijmm.2011.09.006

Kolodziejek, A. M., Schnider, D. R., Rohde, H. N., Wojtowicz, A. J., Bohach, G. A., Minnich, S. A., et al. (2010). Outer membrane protein X (Ail) contributes to Yersinia pestis virulence in pneumonic plague and its activity is dependent on the lipopolysaccharide core length. Infect. Immun. 78, 5233-5243. doi: 10.1128/IAI.00783-10

Li, Y., Cao, S., Zhang, L., Yuan, J., Lau, G. W., Wen, Y., et al. (2016). Absence of TolC impairs biofilm formation in Actinobacillus pleuropneumoniae by reducing initial attachment. PLoS ONE 11:e0163364. doi: 10.1371/journal.pone.0163364

Lloyd, S. J., Ritchie, J. M., Rojas-Lopez, M., Blumentritt, C. A., Popov, V. L., Greenwich, J. L., et al. (2012). A double, long polar fimbria mutant of Escherichia coli O157:H7 expresses Curli and exhibits reduced in vivo colonization. Infect. Immun. 80, 914-920. doi: 10.1128/IAI. 05945-11

Lyhs, U., Ikonen, I., Pohjanvirta, T., Raninen, K., Perko-Makela, P., and Pelkonen, S. (2012). Extraintestinal pathogenic Escherichia coli in poultry meat products on the Finnish retail market. Acta Vet. Scand. 54:64. doi: 10.1186/1751-0147-54-64

Magistro, G., Hoffmann, C., and Schubert, S. (2015). The salmochelin receptor IroN itself, but not salmochelin-mediated iron uptake promotes biofilm formation in extraintestinal pathogenic Escherichia coli (ExPEC). Int. J. Med. Microbiol. 305, 435-445. doi: 10.1016/j.ijmm.2015.03.008

Manges, A. R., and Johnson, J. R. (2012). Food-borne origins of Escherichia coli causing extraintestinal infections. Clin. Infect. Dis. 55, 712-719. doi: $10.1093 / \mathrm{cid} / \mathrm{cis} 502$

Mecsas, J., Welch, R., Erickson, J. W., and Gross, C. A. (1995). Identification and characterization of an outer membrane protein, OmpX, in Escherichia coli that is homologous to a family of outer membrane proteins including Ail of Yersinia enterocolitica. J. Bacteriol. 177, 799-804. doi: 10.1128/jb.177.3.799-804.1995

Meng, X., Liu, X., Zhang, L., Hou, B., Li, B., Tan, C., et al. (2016). Virulence characteristics of extraintestinal pathogenic Escherichia coli deletion of gene encoding the outer membrane protein X. J. Vet. Med. Sci. 78, 1261-1267. doi: 10.1292/jvms.16-0071 
Mika, F., and Hengge, R. (2014). Small RNAs in the control of Rpos, CsgD, and biofilm architecture of Escherichia coli. RNA Biol. 1, 494-507. doi: $10.4161 /$ rna.28867

Miller, V. L., Beer, K. B., Heusipp, G., Young, B. M., and Wachtel, M. R. (2001). Identification of regions of Ail required for the invasion and serum resistance phenotypes. Mol. Microbiol. 41, 1053-1062. doi: 10.1046/j.1365-2958.2001.02575.x

Misra, R., and Reeves, P. R. (1987). Role of micF in the tolC-mediated regulation of OmpF, a major outer membrane protein of Escherichia coli K-12. J. Bacteriol. 169, 4722-4730. doi: 10.1128/jb.169.10.4722-4730.1987

Mitchell, N. M., Johnson, J. R., Johnston, B., Curtiss, R. III, and Mellata, M. (2015). Zoonotic potential of Escherichia coli isolates from retail chicken meat products and eggs. Appl. Environ. Microbiol. 81, 1177-1187. doi: 10.1128/AEM.03524-14

Otto, K., and Hermansson, M. (2004). Inactivation of ompX causes increased interactions of type 1 fimbriated Escherichia coli with abiotic surfaces. J. Bacteriol. 186, 226-234. doi: 10.1128/JB.186.1.226-234.2004

Otto, K., and Silhavy, T. J. (2002). Surface sensing and adhesion of Escherichia coli controlled by the Cpx-signaling pathway. Proc. Natl. Acad. Sci. U.S.A. 99, 2287-2292. doi: 10.1073/pnas.042521699

Pletzer, D., Stahl, A., Oja, A. E., and Weingart, H. (2015). Role of the cell envelope stress regulators BaeR and CpxR in control of RNDtype multidrug efflux pumps and transcriptional cross talk with exopolysaccharide synthesis in Erwinia amylovora. Arch. Microbiol. 197, 761-772. doi: 10.1007/s00203-015-1109-0

Prigent-Combaret, C., Brombacher, E., Vidal, O., Ambert, A., Lejeune, P., Landini, P., et al. (2001). Complex regulatory network controls initial adhesion and biofilm formation in Escherichia coli via regulation of the csgD gene. J. Bacteriol. 183, 7213-7223. doi: 10.1128/JB.183.24.7213-7223.2001

Ren, D., Bedzyk, L. A., Thomas, S. M., Ye, R. W., and Wood, T. K. (2004). Gene expression in Escherichia coli biofilms. Appl. Microbiol. Biotechnol. 64, 515-524. doi: 10.1007/s00253-003-1517-y

Smith, J. L., Fratamico, P. M., and Gunther, N. W. (2007). Extraintestinal pathogenic Escherichia coli. Foodborne Pathog. Dis. 4, 134-163. doi: $10.1089 /$ fpd.2007.0087

Steenackers, H., Hermans, K., Vanderleyden, J., and De Keersmaecker, S. C. (2012). Salmonella biofilms: an overview on occurrence, structure, regulation and eradication. Food Res. Int. 45, 502-531. doi: 10.1016/j.foodres.2011.01.038

Stepanovic, S., Vukovic, D., Dakic, I., Savic, B., and Svabic-Vlahovic, M. (2000). A modified microtiter-plate test for quantification of staphylococcal biofilm formation. J. Microbiol. Methods 40, 175-179. doi: 10.1016/S0167-7012(00)00122-6

Stoorvogel, J., Van Bussel, M. J., and Van De Klundert, J. A. (1991). Biological characterization of an Enterobacter cloacae outer membrane protein (OmpX). J. Bacteriol. 173, 161-167. doi: 10.1128/jb.173.1.161-167.1991

Van Houdt, R., and Michiels, C. W. (2005). Role of bacterial cell surface structures in Escherichia coli biofilm formation. Res. Microbiol. 156, 626-633. doi: 10.1016/j.resmic.2005.02.005

Viveiros, M., Dupont, M., Rodrigues, L., Couto, I., Davin-Regli, A., Martins, M., et al. (2007). Antibiotic stress, genetic response and altered permeability of E. coli. PLoS ONE 2:e365. doi: 10.1371/journal.pone.00 00365

Vogt, J., and Schulz, G. E. (1999). The structure of the outer membrane protein OmpX from Escherichia coli reveals possible mechanisms of virulence. Structure 7, 1301-1309. doi: 10.1016/S0969-2126(00)80063-5

White, A. P., Gibson, D. L., Collinson, S. K., Banser, P. A., and Kay, W. W. (2003). Extracellular polysaccharides associated with thin aggregative fimbriae of Salmonella enterica serovar enteritidis. J. Bacteriol. 185, 5398-5407. doi: 10.1128/JB.185.18.5398-5407.2003

Yang, J. N., Wang, C., Guo, C., Peng, X. X., and Li, H. (2011). Outer membrane proteome and its regulation networks in response to glucose concentration changes in Escherichia coli. Mol. Biosyst. 7, 3087-3093. doi: 10.1039/c1mb05193h

Zgurskaya, H. I., Krishnamoorthy, G., Ntreh, A., and Lu, S. (2011). Mechanism and function of the outer membrane channel TolC in multidrug resistance and physiology of Enterobacteria. Front. Microbiol. 2:189. doi: $10.3389 /$ fmicb.2011.00189

Conflict of Interest Statement: The authors declare that the research was conducted in the absence of any commercial or financial relationships that could be construed as a potential conflict of interest.

Copyright (C) 2018 Li, Huang, Cui, Liu, Hou, Zhang, Liu, Meng and Li. This is an open-access article distributed under the terms of the Creative Commons Attribution License (CC BY). The use, distribution or reproduction in other forums is permitted, provided the original author(s) and the copyright owner are credited and that the original publication in this journal is cited, in accordance with accepted academic practice. No use, distribution or reproduction is permitted which does not comply with these terms. 